\title{
Torsion of paratubal cyst - a rare cause of acute abdominal pain in an adolescent: case report and review of literature
}

\author{
Renuka Malik*, Sonal Gupta
}

Department of Obstetrics and Gynaecology, PGIMER, Dr RML Hospital, New Delhi, India

Received: 16 March 2016

Revised: 04 April 2016

Accepted: 06 April 2016

\author{
*Correspondence: \\ Dr. Renuka Malik, \\ E-mail: renucam@yahoo.co.in
}

Copyright: ( $)$ the author(s), publisher and licensee Medip Academy. This is an open-access article distributed under the terms of the Creative Commons Attribution Non-Commercial License, which permits unrestricted non-commercial use, distribution, and reproduction in any medium, provided the original work is properly cited.

\begin{abstract}
Twisted paraovarian cyst is rare. However, it should be kept as differential diagnosis in any case presenting with pain abdomen. We report a case of a young girl who presented in our OPD with abdominal pain and had twisted paratubal cyst whose torsion had let to spontaneous fimbrial amputation and was managed laparoscopically. 16 years old girl presented in Gynaecology OPD with acute lower abdominal pain with vomiting. Ultrasound done showed a paraovarian cyst on left, 10x7 cm with normal bilateral ovaries. A laparoscopy was done which showed a large gangrenous cyst on left with detached fimbria over it, bilateral ovaries were normal. Laparoscopic cystectomy was done and cyst removed through $10 \mathrm{~mm}$ port. Though benign in nature, rarely paraovarian cyst can undergo torsion causing acute pain and can rarely compromise fertility.
\end{abstract}

Keywords: Paratubal cyst, Paraovarian cyst, Torsion, Acute abdomen

\section{INTRODUCTION}

Para ovarian cyst/fimbrial cyst/paratubal cysts are small, sometimes multiple cysts, may or may not be pedunculated and are seen in $4 \%$ of women. They represent approximately $10 \%$ of adenexal masses. ${ }^{1,2}$ They occur in all age groups, most common in third to firth decade. In paediatric and adolescent population, a much higher incidence of paratubal cysts was reported (7.3\%). Para ovarian cysts lie close to ovary and fimbria. Most of the time they remain small and asymptomatic but sometimes they grow large in size and can undergo complications like infection, hemorrhage and torsion causing acute pain. They can cause secondary torsion of tube, secondary torsion of ovary and rarely become malignant in $2-3 \%$ of cases. ${ }^{1-4}$ Due to their proximity to ovary and fimbria, torsion can compromise ovary or fimbrial integrity as seen in our case. This is a very unusual manifestation but should be kept in mind in women having adenexal masses.

\section{CASE REPORT}

A sixteen year old girl presented on $26^{\text {th }}$ February, 2016 in OPD with chief complaints of pain abdomen and multiple episodes of vomiting of one day duration. The patient was apparently well until 4 months back when she started having episodes of pain confined to lower abdomen, more on left side, severe in intensity, each episode lasting for 2-3 days. The pain had progressed in intensity in the last month and used to occur at more frequent intervals. she was consulting another hospital and also some other clinics during last four months for pain abdomen. The patient also gave history of frequent menstrual cycles with heavy blood flow with dysmenorrhea. Her ultrasound with Doppler done in another hospital, four month back showed a left ovarian cyst $7 \times 4 \mathrm{~cm}$ (Figure 1A and B), with no increased vascularity and patient was advised tumor markers namely CA 125, LDH, AFP and CEA which were all normal. On basis of that, she was advised conservative management. When she kept having pain off and on, she 
consulted another clinic, where an ultrasound report of intestinal duplication cyst was given and patient was advised surgical consultation. A CT scan had been done one month back and was reported as $9.8 \times 7.5 \times 5.4 \mathrm{~cm}$ large unilocular cyst superior to bladder with normal bilateral ovaries with clinical impression of exophytic ovarian cyst with normal bilateral ovaries. This time when she had pain, she reported to our hospital. On examination, patient's general condition was stable, she had mild pallor, was afebrile, her pulse rate was 104/min. regular, blood pressure was 120/80 mm $\mathrm{Hg}$. and her hydration was fair. The per-abdominal examination was normal except tenderness in left iliac fossa, $\mathrm{P} / \mathrm{R}$ was normal. After admission, routine blood investigations were done and were found to be normal. An ultrasound was done with Doppler flow by radiologist who reported a $9.8 \times 7.2 \times 4.4 \mathrm{~cm}$ left paraovarian cyst with no increased vascularity. Patient was prepared for operative laparoscopy. One $10 \mathrm{~mm}$ primary umbilical port and two secondary contra lateral $5 \mathrm{~mm}$ ports were used. Laparoscopy revealed a large $10 \times 8 \mathrm{~cm}$ bluish black paraovarian cyst on left, with normal ovaries. The cyst had fimbria attached to its surface and terminal end of left tube was swollen and blind. Incision was given over the cyst and it was enucleated Controlled suction was done for debuking and it revealed clear fluid. The entire cyst was removed through $10 \mathrm{~mm}$ port, avoiding any spillage (Figure 2A-F). Post op period was uneventful and patient was discharged on third day. Histopathology report of the patient revealed an infarcted cyst lined by low cuboidal epithelium, consistent with torsion.

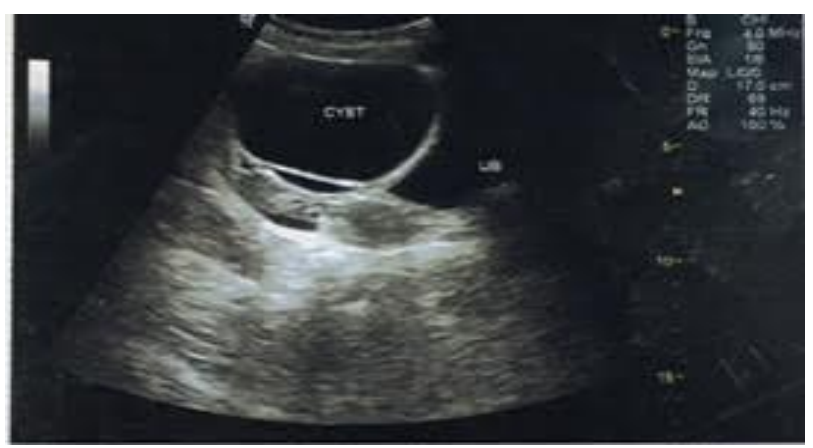

Figure 1A: Ultrasound done four month back.

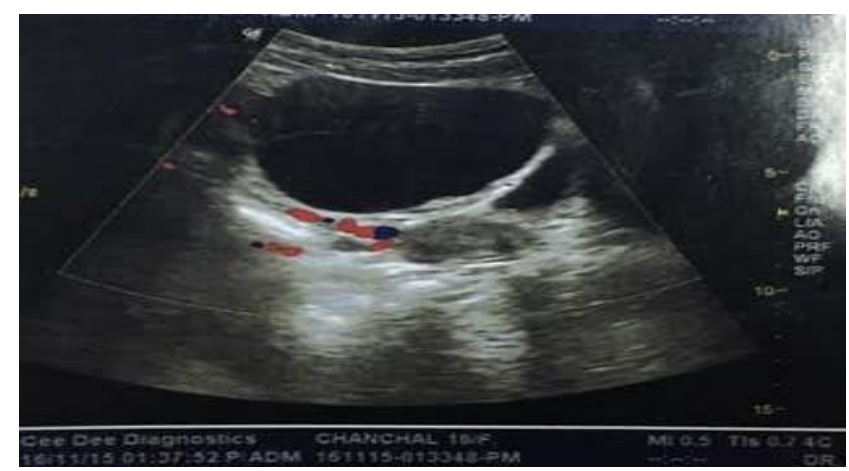

Figure 1B: Doppler done four month back.

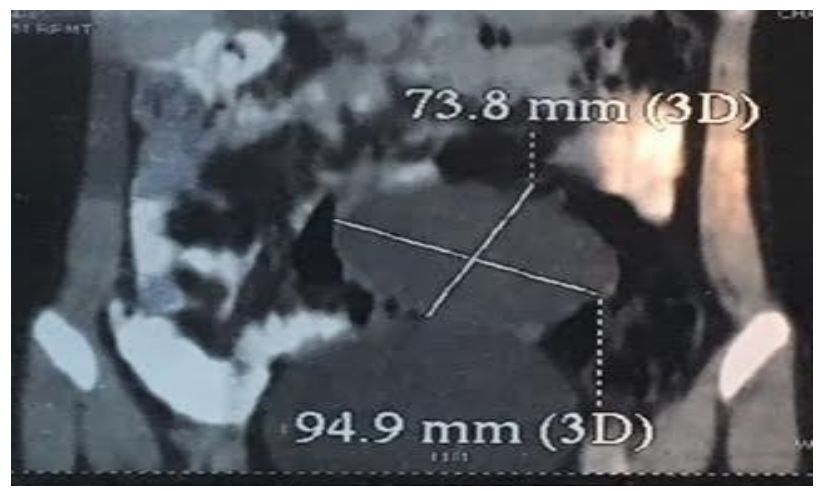

Figure 1C: CT of the cyst.

\section{DISCUSSION}

Para tubal and paraovarian cysts are a relatively common entity, representing $4.7-20 \%$ of all adnexal masses Paraovarian cysts arise from the tissues of the broad ligament, predominantly from mesothelium covering the peritoneum but also from paramesonephric and rarely mesonephric remnants. Para tubal cysts including fimbrial cysts or hydatid cysts of Morgagni are of Mesonephric origin, while paraovarian cysts are of Wolffian or paramesonephric in origin mullerian duct forms the fallopian tube at about 9 weeks of gestation. Multiple invaginations near the ostium of the tube become the fimbriae. Any secondary invagination that does not connect may form a blind sac and enlarge to form a paraovarian cyst the majority of these cysts are small, unilateral, unilocular and benign, though rarely large, bilateral, multicystic and even borderline tumors and carcinomas have been reported in 2-3\%

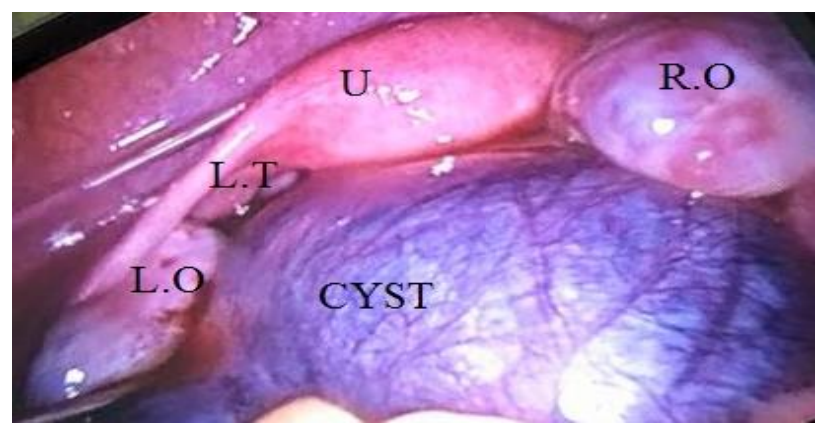

Figure 2A: Operative laparoscopy; Large cyst on left with both ovaries.

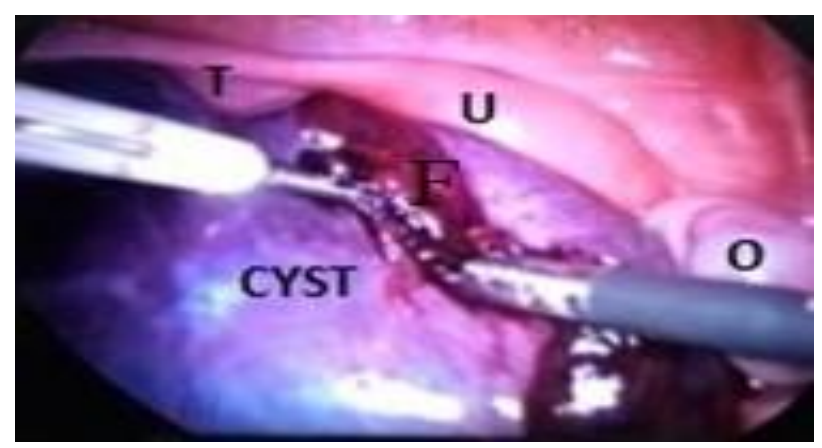

Figure 2B: Operative laparoscopy; Fimbria over cyst. 


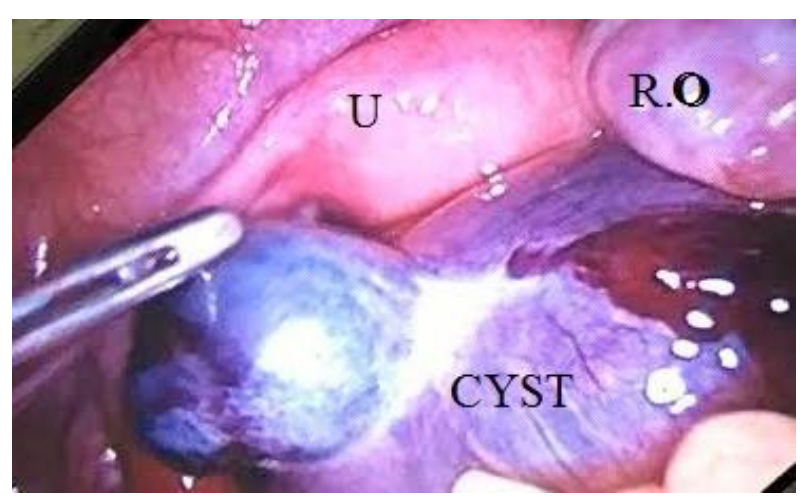

Figure 2C: Operative laparoscopy; Terminal hydrosalpinx.

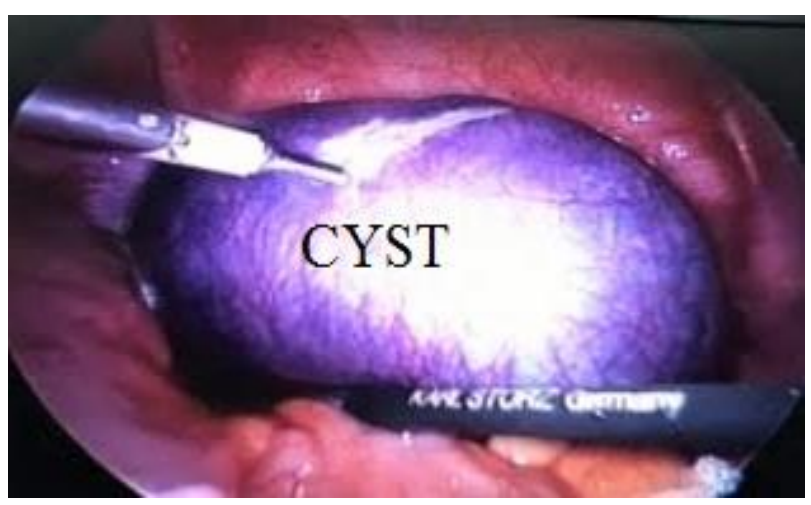

Figure 2D: Operative laparoscopy; Cyst being enucleated.

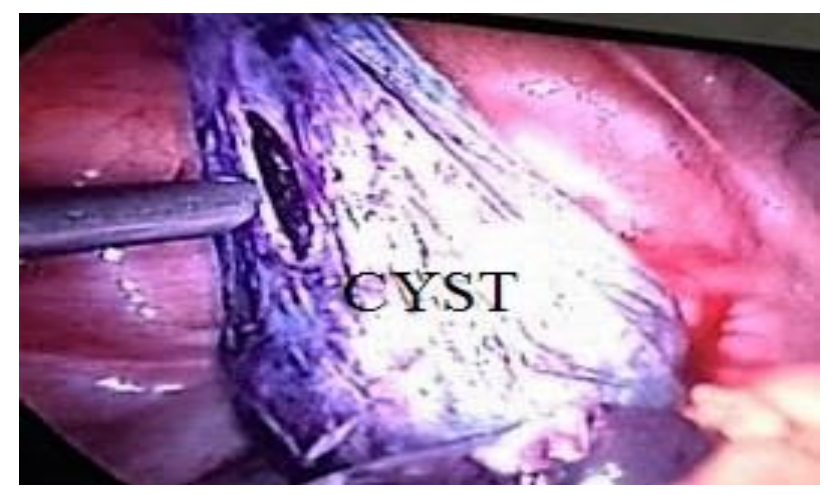

Figure 2E: Operative laparoscopy; Cyst being aspirated.

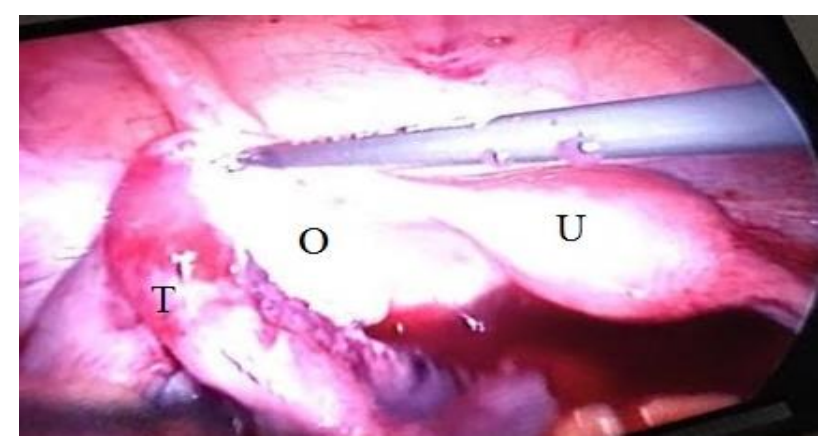

Figure 2F: Operative laparoscopy; Blind terminal left fallopian tube after removal of cyst.
Generally, terminology depends upon the location of cysts. Fimbrial cysts are often tiny multiple and commonly pedunculaed and attached to fimbria. They are twice common in patients with unexplained infertility possibly because causing of interference with tubal pickup. ${ }^{2}$

These masses remain asymptomatic and are often identified incidentally on imaging for other reasons. Additionally, like other adnexal pathology, these cysts can torse (2-16\%), become infected or haemorrhagic, rupture or become malignant. Torsion of the paraovarian cyst is three times more common in pregnant women probably related to rapid growth spurt.

There are reports in literature of their causing secondary torsion of tube and secondary torsion of ovary. ${ }^{1,3-5}$ However, they may also present with pain or pressure symptoms. Few cases are reported in the literature where they exceed $15 \mathrm{~cm}$ of diameter. In the literature, the biggest paraovarian cyst is reported by Letourneuris ${ }^{6}$, a $36 \times 9 \times 25 \mathrm{~cm}$ mass in a 19-year-old girl, which was managed by suprapubic laparotomy following laparoscopy. Another complication of large paratubal cyst reported was hydronephrosis due to their close proximity to tube and ovary, torsion of cyst can compromise ovarian or fimbrial integrity as seen in our case. ${ }^{7}$ This is a very unusual manifestation but should be kept in mind in women having adnexal masses with pain. ${ }^{9}$ In our case, may be the episodes of pain were due to sub-acute torsion which ultimately cut off the blood supply and detached the fimbria.

The initial evaluation of an adnexal mass starts with ultrasonography either transvaginally or transabdominally. The differential diagnosis of paratubal and paraovarian cyst by ultrasonography requires greater awareness and accuracy. The dissociation of the cyst from the ovary when pushing the probe is a useful sign, called as "split sign" for discriminating paraovarian masses. In another study, preoperative diagnosis of PTC was $26.3 \%$ based on ultrasound or computed tomography Grayscale and color Doppler ultrasound for diagnosing paraovarian or PTCs has $99 \%$ specificity but low sensitivity $(14-47 \%)$ by transvaginal ultrasound. The explanation for discordant results of the studies might be due to experience of the examiner and the quality of the ultrasound used. $^{10,11}$ Moreover, serum Ca 125 measurement does not increase the diagnostic accuracy of transvaginal sonography other imaging techniques like computerized tomography and magnetic resonance imaging can also be used in differential diagnosis Demonstration of a normal ovary, separate from the cyst is an important finding. Ultrasound diagnosis can be difficult and it is prudent to do diagnostic laparoscopy in any women with painful large adnexal mass.

Despite the relative frequency of these masses; there is very little data on their management. The American Congress of Obstetricians and Gynaecologists (ACOG) 2007, states that simple adnexal cysts less than $10 \mathrm{~cm}$ 
may be followed safely without surgical intervention, even in postmenopausal women. It also groups paratubal cysts as non-gynaecological adnexal mass. ${ }^{12}$ However, in view of possible complications there should be a low threshold for laparoscopy A study done by Smorgick et al mentions high $\sim 25 \%$ incidence of neoplasm in paraovarian cysts. ${ }^{13} \mathrm{~A}$ high degree of awareness of possible complications by gynaecologists is paramount in avoiding compromising functional integrity of tubes and ovary, because of the proximity of location.

\section{CONCLUSIONS}

There is a need to maintain a high index of suspicion for this uncommon and often difficult to diagnose cause of abdominal pain. Laparoscopy is the gold standard in diagnosing and managing paratubal cysts and their associated complications.

Funding: No funding sources Conflict of interest: None declared

Ethical approval: Not required

\section{REFERENCES}

1. Dotters-Katz SK, James AH, Jaffe TA. Paratubal/Paraovarian Masses: A Study of Surgical and Non-Surgical Outcomes. Med J Obstet Gynecol. 2014;2(1):1019.

2. Riddle N. Fallopian tubes. Pathology Outlines.com, 24 July 2013.

3. Chauhan S, Blacker C. Paratubal cyst: a case report. W V Med J. 2005;101(4):176.

4. Puri M, Jain K, Negi R. Torsion of para-ovarian cyst: a cause of acute abdomen. Indian $\mathrm{J}$ Med Sci. 2003;57:361.
5. Dani A, Gandi SR. Torsion of paraovarian cyst resulting in secondary torsion of ovary. J Evol Med Dent Sci. 2015;4(28):4901-3.

6. Salmon C, Tornos C, Chi DS. Borderline endometrioid tumor arising in a paratubal cyst: a case report. Gynecol Oncol. 2005;97(1):263-5.

7. Letourneur B, Grandjean S, Richard P, Parant O. Management of a giant paraovarian cyst. Gynecol Obstet Ferti. 2006;134:239-41.

8. Leanza V, Coco L, Pafumi C, Ciotta L. Laparoscopic removal of a giant paratubal cyst complicated by hydronephrosis. G Chir. 2013;34:323-5.

9. Kiseli M, Caglar GS, Cengiz SD, Karadag D, Yılmaz MB. Clinical diagnosis and complications of paratubal cysts: review of the literature and report of uncommon presentations. Arch Gynecol Obstet. 2012;285(1):2304-8.

10. Muolokwu E, Sanchez J, Bercaw JL, SangiHaghpeykar H, Banszek T, Brandt ML. The incidence and surgical management of paratubal cysts in a pediatric and adolescent population. J Ped Surg. 2011;46(11):2161-3.

11. Savelli L, Ghi T, De Iaco P, Ceccaroni M, Venturoli S, Cacciatore B. Paraovarian/paratubal cysts: comparison of transvaginal sonographic and pathological findings to establish diagnostic criteria. Ultrasound Obstet Gynecol. 2006;28:330-4.

12. American college of obstetricians and gynecologists. ACOG Practice Bulletin. Management of adnexal masses. Obstet Gynecol. 2007;110(1):201-14.

13. Smorgick N, Herman A, Schneider D, Halperin R, Pansky M. Paraovarian cysts of neoplastic origin are underreported. J Soc Laparoendoscopic Surg. 2009;13(1):22-6.

Cite this article as: Malik R, Gupta S. Torsion of paratubal cyst - a rare cause of acute abdominal pain in an adolescent: case report and review of literature. Int J Reprod Contracept Obstet Gynecol 2016:5:1625-8. 